\title{
Influence of a Uniform Driving Force on Tracer Diffusion in a One-Dimensional Hard-Core Lattice Gas
}

\author{
R. Kutner ${ }^{1,2}$ and H. van Beijeren ${ }^{3}$
}

Received November 1, 1984; revised December 20, 1984

\begin{abstract}
The influence of a uniform driving force on tracer diffusion is investigated for a one-dimensional lattice gas where particles jump stochastically to unoccupied neighboring sites. A new, simple calculation is presented for the diffusion coefficient of a tracer particie with respect to its average drift, obtained recently by rigorous methods by De Masi and Ferrari. A theoretical expression describing the tracer particle mean square displacement approximately for all times is derived and found to be in excellent agreement with the results of Monte Carlo simulations.
\end{abstract}

KEY WORDS: Tracer diffusion; lattice gas models; excess noise.

\section{INTRODUCTION}

In the present paper we consider tracer diffusion in a one-dimensional lattice gas model in the presence of a driving force. We develop a theory for the mean square displacement of a tracer particle as a function of time by the methods of Ref. 1, which we will refer to as I, and compare its predictions to the results of Monte Carlo simulations.

The model is defined in the following way: A one-dimensional periodic chain of period $N$ is occupied with $N_{p}$ particles, hence the concentration $c$ equals $N_{p} / N$. Double occupancy of sites is forbidden. The particles may only jump to unoccupied neighboring sites, with jump rates $\Gamma_{+}$and $\Gamma_{-}$in

\footnotetext{
${ }^{I}$ Institut für Festkörperforschung, der Kernforschungsanlage Jülich, D-5170 Jülich, West Germany.

${ }^{2}$ Institute of Experimental Physics, University of Warsaw, Hoża 69, 00-681 Warszawa, Poland.

${ }^{3}$ Institut für Theoretische Physik A, Rheinisch-Westfälische Technische Hochschule Aachen, D-5100 Aachen, West Germany.
} 
and against the direction of the driving force, respectively. The jumps are assumed to occur instantaneously and the influence of the force is accounted for entirely by the difference between $\Gamma_{+}$and $\Gamma_{-}$. Note that this model satisfies the so-called single filing constraint ${ }^{(2)}$ : particles cannot pass each other.

If configurations are characterized by specifying the occupied sites (hence ignoring the identity of the particles) the stationary state simply gives equal weight to all allowed configurations. The reason is that there are always equally many configurations from which a given configuration can be gained as to which it can be lost, by a jump in a given direction. ${ }^{(3)}$

In the absence of a driving force the mean square displacement of a tagged particle in this model increases proportionally to the square root of time ${ }^{(1,4-8)}$ in the limit $N \rightarrow \infty$, hence it seems of interest ${ }^{(9,10)}$ to investigate how the mean square of the displacement as measured relative to the average displacement, depends on time when a driving force is present. It was in fact proven recently ${ }^{4}$ that for long times the tagged particle behaves diffusively and the diffusion constant was computed exactly. ${ }^{(9)}$ In Section 2 we propose a theory for the displacement at all times and in Section 3 we present the results of Monte Carlo simulations and compare these to our theory. In Section 4 we discuss our results and make some concluding remarks.

\section{THEORY}

The quantities to be considered here are the mean square displacement $\Delta(t)$ of a tagged particle with respect to its average drift and its velocity autocorrelation function $C(t)$, defined, respectively, as

$$
\Delta(t)=\left\langle[x(t)-x(0)]^{2}\right\rangle-\langle x(t)-x(0)\rangle^{2}
$$

and

$$
C(t)=\langle v(t) v(0)\rangle-\langle v(t)\rangle\langle v(0)\rangle
$$

Here $x(t)$ and $v(t)$ denote the position and velocity of the tagged particle at time $t$ and the brackets denote an average both over the stationary initial distribution and over all realizations of the stochastic hopping process with their proper weights. The quantities $\Delta(t)$ and $C(t)$ are related to each other through the equation ${ }^{(1)}$

$$
\frac{d^{2} \Delta(t)}{d t^{2}}=2 C(t)
$$

${ }^{4}$ C. Kipnis, quoted in Ref. 9. 
Denoting the Laplace transform of $C(t)$ as $\widetilde{C}(s)$ we obtain the frequencydependent tracer diffusion coefficient $D_{\mathrm{tr}}(\omega)$ as

$$
D_{\text {tr }}(\omega)=\operatorname{Re} \widetilde{C}(i \omega)
$$

Its zero frequency limit $D_{\mathrm{tr}}$ is given equivalently by the Einstein relation

$$
D_{\mathrm{tr}}=\lim _{t \rightarrow \infty} \Delta(t) / 2 t
$$

In I an approximate but accurate method was developed for calculating the velocity autocorrelation function for the case $\Gamma_{+}=\Gamma$. It can be applied without any problems to the case where the two jump rates are different.

For a given realization of the hopping process the tracer particles will make jumps $u_{j}$ with $u_{j} \in\{1,-1\}$ (so we set the lattice spacing equal to unity) at time instants $t_{j}(j=0,1,2, \ldots)$, where we require $0 \leqslant t_{0}<t_{1}<\cdots$. Then the (generalized) velocity of the tracer particle is given as

$$
v(t)=\sum_{j=0}^{\infty} u_{j} \delta\left(t-t_{j}\right)
$$

Inserting this expression into (2) one immediately sees that in order to obtain a nonzero contribution to $C(t)$ one must have $t_{0}=0$, so the first jump of the tagged particle has to occur at $t=0$. As in I, we may call the vacancy with which the tagged particle exchanges at $t=0$ the special vacancy and the cluster of vacancies to which it belongs the special vancancy cluster. As in I the dynamics of the special vacancy is defined such, that it may exchange positions with a particle only if no other vacancies are present in the special vacancy cluster and then it does so with the given jump rates $\Gamma_{+}$or $\Gamma_{-}$. In this way the dynamics of the system disregarding the jumps of the special vacancy, would be exactly the same as though the special vacancy were not there at all. Also, if just after the initial jump one takes out the site with the special vacancy, the remaining configuration is stochastically distributed according to the stationary state for a system with $N_{p}$ particles and $N-1$ sites. Hence the average velocity of the tagged particle at time $t$ due to exchanges with nonspecial vacancies, given a jump occurred at $t=0$, in the thermodynamic limit is the same as in the stationary state; it is the constant drift velocity

$$
v_{\mathrm{dr}}=\langle v(0)\rangle=\langle v(t)\rangle=(1-c)\left(\Gamma_{+}-\Gamma_{-}\right)
$$

It then follows that $C(t)$ is entirely due to exchanges between the tagged 
particle and the special vacancy. The initial exchange at $t=0$ gives rise to a contribution $^{(1)}$

$$
C^{(0)}(t)=\left(\Gamma_{+}+\Gamma_{-}\right) \delta(t)
$$

or, after changing to $\widetilde{C}(s)$ in the Laplace domain,

$$
\widetilde{C}^{(0)}(s)=\frac{1}{2}\left(\Gamma_{+}+\Gamma_{-}\right)
$$

It is interesting to consider the case of an infinite driving force. There the initial jump of the tagged particle is in the field direction and after this initial jump no further exchanges between tagged particle and special vacancy are possible. Hence in this case $C(t)$ is identically zero for $t>0$ and the frequency dependent coefficient of tracer diffusion, given by the real part of $\widetilde{C}(i \omega)$, is just a constant. This implies that mean field theory, ${ }^{(11)}$ which approximates $D_{\mathrm{tr}}$ by $\widetilde{C}^{(0)}$, is correct in this limit and that the Bardeen-Herring correlation factor ${ }^{(12)}$ which can be defined as the ratio of the actual tracer diffusion coefficient and its mean field value, becomes equal to unity.

For a general driving force the zero-frequency tracer diffusion coefficient can still be obtained exactly. One only needs to observe that the special vacancy, as seen from the tagged particle, always drifts off in the direction opposite to the field. Hence, if the initial jump of the tagged particle is in the field direction, it is always followed by an even number (which may be 0 ) of further exchanges between special vacancy and tagged particle, before finally the special vacancy drifts off. The contributions of these exchanges to the integral of the velocity autocorrelation function alternatingly are $-(1-c) \Gamma_{+}$and $(1-c) \Gamma_{+}$, hence they always add up to zero. On the other hand, if the initial jump was against the field direction it is always followed by an odd number of subsequent exchanges, the first one of which contributes $-(1-c) \Gamma_{-}$, while the sum of the other ones adds up to zero again. Adding this additional contribution to the initial contribution given in (9) one finds

$$
D_{\mathrm{tr}}=\lim _{s \rightarrow 0} \tilde{C}(s)=\frac{1}{2}(1-c)\left(\Gamma_{+}-\Gamma_{-}\right)
$$

A rigorous derivation of this result has been given by De Masi and Ferrari. ${ }^{(9)}$

The full time behavior of $C(t)$ for arbitrary driving field is not so easy to calculate exactly. However, as was done in I, one can approximate the special vacancy dynamics by making the following three assumptions about the behavior of the special vacancy cluster: 
(1) Just after a jump of the special vacancy the probability that the special vacancy cluster contains $m$ vacancies, including the special one equals $c(1-c)^{m-1}(m=1,2, \ldots)$.

(2) A special vacancy cluster of size $m$ has a constant rate $(1-c)\left(\Gamma_{+}+\Gamma_{-}\right)$for growth to size $m+1$.

(3) A special vacancy cluster of size $m$ has a constant rate $\left(\Gamma_{+}+\Gamma_{-}\right)$for shrinking to size $m-1$.

As discussed in I the assumptions (1) and (2) are based on the stationary state values and they ignore memory effects.

Under the assumptions given above the special vacancy, as seen from the tagged particle, describes a biased continuous time random walk, with waiting time distributions $(1-p) \psi(t)$ and $p \psi(t)$, where $p=\Gamma_{+} /\left(\Gamma_{+}+\Gamma_{-}\right)$, for jumps in and against the field direction, respectively. The Laplace transform $\widetilde{\psi}(s)$ of $\psi(t)$ can be taken from (I.2.10) as

$$
\widetilde{\psi}(s)=\frac{c v}{[1-\widetilde{X}(s)](2-c)(v+s)-(1-c) v}
$$

with, however, $v=\left(\Gamma_{+}+\Gamma_{-}\right)(2-c)$ and $\tilde{X}(s)$ given again by (I.2.12) in the form

$$
\tilde{X}(s)=\frac{1}{2}-\frac{v}{2(s+v)(2-c)}\left\{c^{2}+(2-c)^{2}\left[\frac{2 s}{v}+\left(\frac{s}{v}\right)^{2}\right]\right\}^{1 / 2}
$$

Now if we define $P(m \mid n, t)$ as the probability density for the special vacancy, having arrived at site $n$ at time $t=0$, to jump so site $m$ at the instant $t,{ }^{5}$ its Laplace transform can be found, e.g., by the calculation given in the Appendix of $\mathrm{I}$, to be

$$
\begin{aligned}
& \tilde{P}(m \mid n, s)=\left[\frac{2(1-p) \widetilde{\psi}(s)}{1+\left[1-4 p(1-p) \tilde{\psi}^{2}(s)\right]^{1 / 2}}\right]^{m-n} \frac{1}{\left[1-4 p(1-p) \tilde{\psi}^{2}(s)\right]^{1 / 2}}, \\
&=\left[\frac{2 p \tilde{\psi}(s)}{1+\left[1-4 p(1-p) \tilde{\psi}^{2}(s)\right]^{1 / 2}}\right]^{n-m} \frac{1}{\left[1-4 p(1-p) \tilde{\psi}^{2}(s)\right]} \\
& m \leqslant n
\end{aligned}
$$

Now it is simple to calculate the Laplace transform of the velocity

\footnotetext{
${ }^{5}$ This means that after a time $t$ the special vacancy has jumped over a net number of $|n-m|$ particles, either in the field direction $(n \geqslant m)$ or in the opposite direction $(n \leqslant m)$.
} 
autocorrelation function. Under the approximations made $\widetilde{C}(s)$ can be written as

$$
\begin{aligned}
\tilde{C}(s)= & \tilde{C}^{(0)}+(1-c) \Gamma_{+}\{\widetilde{P}(0 \mid 1, s) p \widetilde{\psi}(s)-\widetilde{P}(0 \mid 0, s)(1-p) \widetilde{\psi}(s)\} \\
& +(1-c) \Gamma_{-}\{\tilde{P}(0 \mid-1, s)(1-p) \widetilde{\psi}(s)-\widetilde{P}(0 \mid 0, s) p \widetilde{\psi}(s)\}
\end{aligned}
$$

For understanding this expression: e.g., the term $(1-c) \Gamma_{+} \widetilde{P}(0 \mid 1, s) p \widetilde{\psi}(s)$ describes the Laplace transform of the probability density that the special vacancy, after making an initial jump against the field direction [with probability density $\left.(1-c) \Gamma_{+}\right]$performs a random walk bringing it one position to the right [with probability density $P(0 \mid 1, t)$ ], convoluted with the probability density for a final jump to the left [with probability density $p \psi(t)]$ occuring at time $t$. This term yields a positive contribution to the velocity autocorrelation function, since the initial and the final jump are in the same direction. The other terms can be understood in similar ways. Inserting Eqs. (11-13) into (14) one obtains the final result

$$
\widetilde{C}(s)=\frac{1}{2}(1-c)\left(\Gamma_{+}+\Gamma_{-}\right) \frac{1-4 p(1-p) \tilde{\psi}(s)}{\left[1-4 p(1-p) \tilde{\psi}^{2}(s)\right]^{1 / 2}}
$$

In the asymptotic region $c \rightarrow 1$ the inverse Laplace transform of this equation can be obtained explicitly as

$$
\begin{aligned}
C(t)= & (1-c)\left(\Gamma_{+}+\Gamma_{-}\right)^{2}\left[\delta(\tau)+\sqrt{p(1-p)} e^{-\tau}\right. \\
& \left.\times\left\{I_{1}(2 \tau \sqrt{p(1-p)})-I_{0}(2 \tau \sqrt{p(1-p)})\right\}\right]
\end{aligned}
$$

where $I_{n}$ denotes a Bessel function of imaginary argument and $\tau=\left(\Gamma_{+}+\Gamma_{-}\right) t$ is a dimensionless time unit. In the general case $C(t)$ has to be obtained by a numerical inverse Laplace transform.

\section{NUMERICAL SIMULATIONS AND RESULTS}

The hopping process with different jump rates $\Gamma_{+}$and $\Gamma_{-}$was simulated by a Monte Carlo procedure that has been described comprehensively in Refs. 11 and 14 treating tracer diffusion in an fcc lattice and in a model consisting of two coupled lines. Here we discuss some characteristic features and modifications for the model discussed in this paper.

For the size of the lattice we chose $N=200000$, which means that over the time ranges considered finite size effects on averages are completely unimportant, and we used periodic boundary conditions. Initial configurations were generated by occupying the sites randomly with average concentration $c$. The values of $c$ we investigated are $c=0.2,0.5$, 
and 0.8. In order to achieve better statistics for $c>0.5$ the following modification was made: in each Monte Carlo step a vacancy is selected at random (instead of a particle, as was done in previous versions) and with probability $p$, respectively $(1-p)$, it exchanges positions with the content of the neighboring site to its left, respectively, its right. In this way two vacancies may be exchanged, but this does not influence the dynamics of the particles. The initial and actual positions of both particles and vacancies are stored, hence one easily obtains quantities like $\Delta(t)$. For $c<0.5$ particles instead of vacancies are chosen at random, but now an attempted jump is actually performed only if the exchange is to take place with a vacancy. It is clear that the ratio between the efficiency rates of the two strategies (the fractions of attempted jumps that exchange a particle and a vacancy) equals $(1-c) / c$ so that indeed for $c>0.5$ the first strategy is preferable and for $c<0.5$ the second one. Further, in the first procedure the discrete time $\tau$ is measured in Monte Carlo steps per vacancy $(M c s / v)$, i.e., $\tau=N_{\text {st }} /\{(1-c) N\}$ were $N_{\text {st }}$ is the number of Monte Carlo steps, and in the second procedure it is measured in Monte Carlo steps per particle, i.e., $\tau=N_{\text {st }} /\{c N\}$. In both cases the definition of $\tau$ coincides with the one given in the preceding section, since the average number of jumps exchanging a particle and a vacancy per unit of time equals $c(1-c) N$ in either case.

To compute the quantity $\Delta(t)$ we averaged in each case over all particles and over a few different runs. In this way for all values of $c$, $t$, and $\alpha$, defined as the jump rate ratio $\Gamma_{+} / \Gamma_{-}$, that we investigated, the relative statistical spread in $\Delta(t)$ was found to be less than $0.5 \%$.

In Fig. 1 we display the results of our simulations together with our

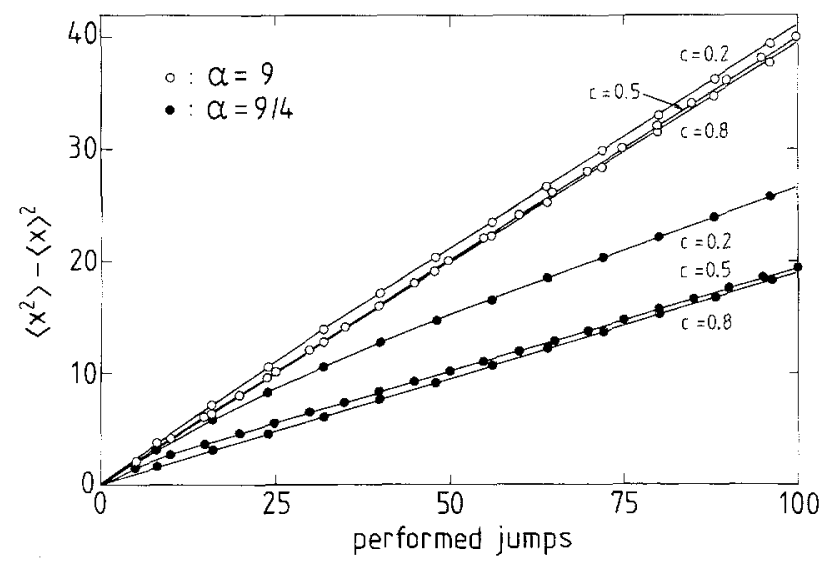

Fig. 1. The dispersion in the mean square displacement of a tracer particle with respect to its average drift is plotted for two different values of the ratio $\alpha=\Gamma_{+} / \Gamma_{-}$and the concentration $c$, as a function of $(1-c)\left(\Gamma_{+}+\Gamma_{-}\right) t$. The solid lines give the theoretical values and the dots our Monte Carlo results. 
theoretical predictions. The latter were obtained by applying an inverse Laplace transform by means of the fast routine of Honig and Hirdes ${ }^{(13)}$ to the quantity $2 \widetilde{C}(s) / s^{2}$, with $\widetilde{C}(s)$ given by (15). The dispersion in the mean square displacement is plotted as a function of $(1-c) \tau$, that is, the time unit is chosen to be the average jumping time of a particle. One sees that the agreement between simulation results and theory is quite satisfactory.

\section{DISCUSSION}

As discussed in Section 2 and Ref. 9 the dispersion of the tracer particle mean square displacement in the presence of a driving force becomes a linear function of time for large times. Plotting the dispersion as a function of $(1-c) \tau$ one finds the asymptotic slope as a function of the jump rate ratio $\alpha=\Gamma_{+} / \Gamma_{-}$to be given by $(\alpha-1) /(\alpha+1)$ independently of $c$, as can be clearly seen in Fig. 1 . Notice that with increasing $\alpha$ the curves for different $c$ come to lay closer together, in accordance with the fact that in the limit $\alpha \rightarrow \infty \Delta(t)$ becomes linear in time for all times; this is an immediate consequence of $\widetilde{C}(s)$ becoming independent of $s$ in this limit.

From (15) one readily finds that for $\alpha \neq 1$ the velocity autocorrelation function decays exponentially for long times, in contrast to the case $\alpha=1$ where the velocity autocorrelation function exhibits a long time tail behavior proportional to $t^{-3 / 2}$. This latter behavior can be explained in a more general theoretical framework alternatively on the basis of mode coupling theory ${ }^{(15)}$ or by a simple argument given by Alexander and Pincus. $^{(8)}$ One would like to understand why these theories would not predict the same type of behavior in the presence of a driving force. The explanation turns out to be rather simple. Mode coupling theory explains the long time tail on the basis of a coupling between a bulk diffusion mode and a tracer diffusion mode. In the presence of a driving force these modes have different drift velocities, namely, $(1-c)\left(\Gamma_{+}-\Gamma_{-}\right)$for the tracer mode and $(1-2 c)\left(\Gamma_{+}-\Gamma_{-}\right)$for the bulk mode ${ }^{(16)}$ (This, incidentally, is the drift velocity of the special vacancy in an inertial reference frame). Hence the two modes drift apart and as a result the correlation between them decays exponentially. Similarly, the argument of Alexander and Pincus is based on the observation that locally the gradient of the displacement of tracer particles from their average positions is to leading order proportional to the fluctuation in the bulk density. In the presence of a driving force one has to consider this relation in a reference frame moving with the tracer particle drift velocity. In such a reference frame the bulk mode drifts with velocity $-c\left(\Gamma_{+}-\Gamma_{-}\right)$, which leads again to an exponential decay of the local fluctuation of the bulk density (which in our case may be interpreted as the local special vacancy density). 
Finally we want to remark that the effects reported here may be observable in real experiments with the aid of magnetic tagging techniques. ${ }^{(17-19)}$ With this method for instance ionic mobilities can be determined over a wide range of values.

\section{ACKNOWLEDGMENTS}

The authors thank K. W. Kehr for discussions and H.v.B. thanks the I.H.E.S. at Bures-sur-Yvette for its hospitality while part of this paper was prepared. Partial support to R.K. was given by the Polish Ministry of Higher Education, Science and Technology, Project M.R.I. 5/K-8.6.01.

\section{REFERENCES}

1. H. van Beijeren, K. W. Kehr, and R. Kutner, Phys. Rev. B 28:5711 (1983).

2. K. Heckmann, in Passive Permeability of Cell Membranes, Biomembranes, Vol. 3, F. Kreuzer and J. F. G. Slegers, eds. (Plenum Press, New York, 1972), p. 127.

3. H. van Beijeren and L. S. Schulman, Phys. Rev. Lett. 53:806 (1984).

4. T. E. Harris, J. Appl. Prob. 2:323 (1965).

5. F. Spitzer, Adv. Math. 5:246 (1970).

6. P. M. Richards, Phys. Rev. B 16:2011 (1978).

7. P. A. Fedders, Phys. Rev. B 17:40 (1978).

8. S. Alexander and P. Pincus, Phys. Rev. B 18:2011 (1978).

9. A. de Masi and P. A. Ferrari, Self-diffusion in one dimensional lattice gases in the presence of an external field, J. Stat. Phys. 38:603 (1985).

10. H. Spohn, Excess noise for a lattice gas model of a resistor, Z. Phys. B 57:255 (1984).

11. K. W. Kehr, R. Kutner, and K. Binder, Phys. Rev. B 23:4931 (1981).

12. J. Bardeen and C. Herring, in Imperfections in Nearly Perfect Crystals, W. Shockley, ed. (Wiley, New York, 1952).

13. G. Honig and U. Hirdes, J. Comput. Appl. Math. 10:113 (1984).

14. R. Kutner, H. van Beijeren, and K. W. Kehr, Phys. Rev. B 30:1984.

15. H. van Beijeren, J. Stat. Phys. 32:399 (1984).

16. S. Katz, J. L. Lebowitz, and H. Spohn, Phys. Rev. B 28:1655 (1983) (see Appendix A).

17. U. V. Alpen, H. Schultz, G. H. Talal, and H. Bohm, Solid State Commun. 23:911 (1977).

18. W. L. Roth, F. Reidinger, and S. La Placa, in Superionic Conductors, C. D. Mahan and W. L. Roth, eds. (Plenum Press, New York, 1976).

19. R. D. Hogg, S. P. Vernon, and V. Jaccarino, Phys. Rev. Lett. 39:481 (1977). 